\title{
E30 MACRODISPERSION APPROACH FOR UPSCALING OF TWO-PHASE, IMMISCIBLE FLOW IN HETEROGENEOUS POROUS MEDIA.
}

\begin{abstract}
This paper focuses on upscaling of immiscible flow in porous media. It is currently admitted that classical pseudoization methods suffer some limitations, mainly due to the fact that numerical and physical coarsening are coupled within this framework. In order to overcome this problem, we look at the analytical form of upscaled transport equations, reserving the grid blocks coarsening to a subsequent step.

Our study confirms that a dispersive flux must be included in the large scale equations, to correctly model the evolution of mean saturation profiles, as predicted by a macrodispersion approach already used by some authors. The validity of this approach is confirmed by numerical simulations of two-phase immiscible flow in heterogeneous porous media (lognormal absolute permeability fields, uniform relative permeabilities).

For favorable mobility ratios, the smeared frontal zone tends to stabilize and become stationary throughout time, depending on the macrodipersive flux term. A finite size transition zone appears, which replaces the sharp water/oil front. Behind this zone, we recover the hyperbolic profile described by the theory of Buckley and Leverett. These observations were used to fit a dispersion coefficient, using a semi-analytical asymptotic solution of two-phase immiscible flows with dispersion. Results were compared with previous theoretical predictions. In the unfavorable mobility ratio cases, a continuous convective stretching of profiles occurs, even in the frontal zone, and transition from local scale to large scale description should involve both a modification of the convective flux and the introduction of a dispersive term.
\end{abstract}

Such a difference in behavior was not captured by previous studies, and especially the emergence of a finite size transition zone. This last fact cannot be understood within the Lagrangian framework without invoking nonlinear effects.

\section{Introduction}

Current numerical models of reservoirs can contain several millions gridblocks, leading to finescale flow simulations often exceeding classical computational resources. Upscaling fine-scale heterogeneity is then used to reduce the number of gridblocks in the simulation model. This problem is particularly challenging when dealing with multiphase flows, and has been widely investigated for several decades. So far, two major approaches have been undertaken.

The first approach, known as pseudoization, consists in averaging several sub-gridblocks properties in a single coarse block, assuming that the type of flow equations remains the same from the smaller to the larger scale. This assumption leads to effective parameters depending on

$8^{\text {th }}$ European Conference on the Mathematics of Oil Recovery - Freiberg, Germany, 3 - 6 September 2002 
the boundary conditions and the position of the coarse block in the whole grid. These parameters also take into account the effects of numerical dispersion associated with the coarsening of gridblocks size. Except in the vertical equilibrium case, this approach requires a full fine-scale flow simulation on at least a representative part of the reservoir. This leads to a lot of theoretical and practical problems, although pseudoization is still the most widely used approach in problems of practical interest. Further details and references can be found in [1].

The second method searches for a homogenized description of flow at a larger scale. The aim is to find the upscaled form of flow equations and to derive intrinsic flow parameters at the desired scale by solving a purely physical problem. The numerical problem of coarsening the gridblocks size is kept for a subsequent step. Although this approach has been widely investigated in solute or miscible transport theory, it has been only recently undertaken for two-phase flow problems. Based on numerical simulation results, our study describes several patterns affecting two-phase immiscible flows in heterogeneous porous media in order to understand the mechanisms to be included in a homogenization approach.

\section{Simulations and results}

\subsection{Experiences}

Flow simulations were performed on two-dimensional realizations of isotropic lognormal absolute permeability fields $K(\boldsymbol{x})$ with known variogram, mean and variance on a square $300 * 300$ grid. Each gridblock has a size of $1 \mathrm{~m} * 1 \mathrm{~m}$. Exponential and spherical variograms were tested, with a constant correlation length of $6 \mathrm{~m}$. The geometrical mean of absolute permeability is $50 \mathrm{mD}$, the variance of $\ln (K)$ evolves in the range from 0.1 to 2.0 .

The porosity was kept constant and the relative permeability curves are of Corey-type with an exponent equal to 2 and no residual saturation : $k_{r w}(S)=S^{2}$ for the injected fluid and $k_{r n}(S)=(1-S)^{2}$ for the displaced fluid, where $S$ is the saturation of the injected fluid. Gravity and capillary effects are supposed to be negligible. Two opposite sides have a zero-flux condition, while the inlet face have a constant flux condition : $100 \%$ water is injected at fixed rate. The main flow is in the positive $\mathrm{x}$-direction. We also impose a constant pressure along the side $x=300 \mathrm{~m}$. The viscosity ratio $M$ between the fluid in place (2) and the injected one (1) varies from 0.1 to 10 . Simulations were performed on both a streamlines-based simulator and a classical simulator. Results from those two simulators show good comparison.

\subsection{Saturation maps}

The saturation maps obtained show two major behaviors, depending on the viscosity ratio. The difference appears when focusing on the front line $x(y, t)$, defined such that $S_{l}(x(y, t))=S_{f}$, where $S_{f}$ is the saturation of the Buckley-Leverett front (homogeneous media with the same $\mathrm{k}_{\mathrm{r}}$ curves). Across this line, the saturation jumps from a completely oil saturated zone to the flooded zone where saturation grows slowly from $S_{f}$ to 1 .

When the viscosity ratio is sufficiently low, the front line is slightly deformed by heterogeneity around a mean position $\bar{x}$ (figure 1a). This mean position corresponds to the theoretical position of the Buckley-Leverett front in a homogeneous medium flooded at the same rate. The variance of the line around $\bar{x}$, defined by :

$$
\sigma_{x}^{2}\left(t, S=S_{f}\right)=\frac{1}{L_{Y}} \int_{0}^{L_{Y}}(x-\bar{x})^{2} d y
$$


rapidly reaches a maximum (figure 1c): while flooding, fingers induced by heterogeneity can't grow and the front line "oscillates" around $\bar{x}$ within a limited width. Hence, the displacement process remains stable. On the other hand, when the viscosity ratio is high, fingers induced by heterogeneity are continuously stretched (figure $1 \mathrm{~b}$ ). There is no stabilization of the front line: its variance grows continuously with time (figurelc).

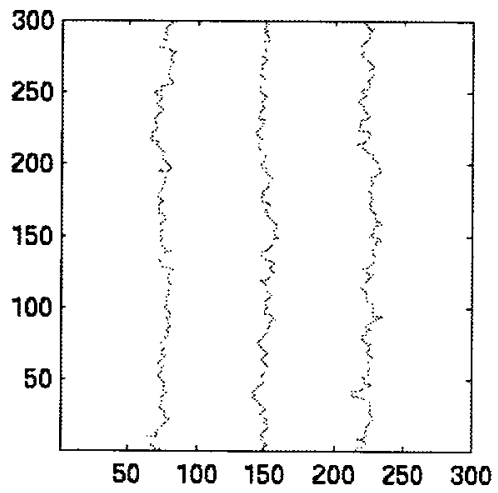

(a)

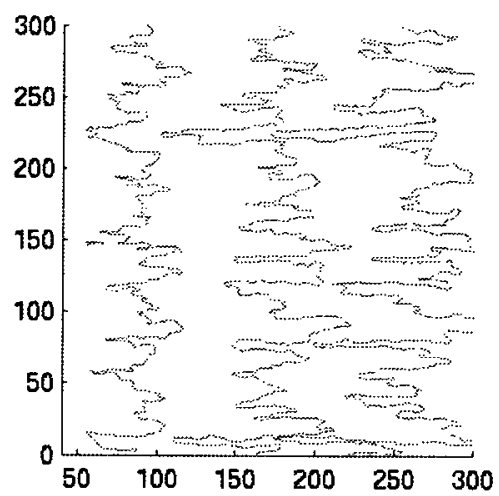

(b)

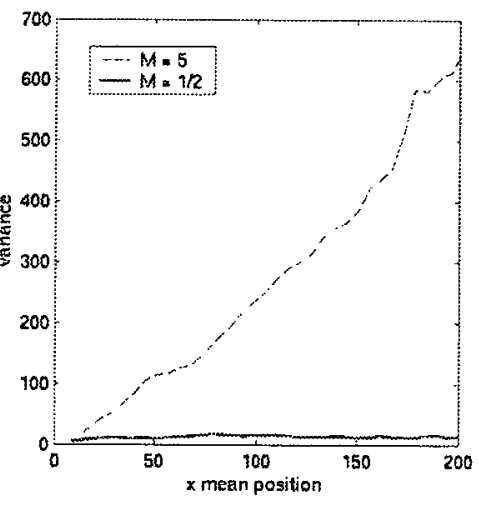

(c)

Figure 1: Evolution of the front line. (a) Stable case. $M=1 / 2, \sigma_{\ln (K)}=0.4$ (b) Unstable case. $M=5$, $\sigma^{2}{ }_{i n(K)}=0.4$ (c) Evolution of the variance of the front line while flooding.

The difference between these two cases is related to viscous forces that tend to stabilize or destabilize the front when a perturbation appears, a mechanism known as Saffman-Taylor instability. The analysis of this phenomenon in homogeneous media for two-phase immiscible flows was studied by, among others, Hagoort [2], who showed that the instability appears when the mobility ratio $M_{f}$ evaluated across the front is larger than one.

\subsection{Mean saturation profiles}

We will now analyze the results in term of mean saturations, averaged along the orthogonal direction to the main flow :

$$
\bar{S}=\frac{1}{L_{Y}} \int_{0}^{L_{Y}} S(x, y, t) d y
$$

This mean saturation corresponds to the actual measures available at a well or at the outlet side of a core. This definition allows the construction of mean saturation profiles along the flow direction, in order to describe the equivalent unidirectional flow to be modeled after homogenization. At this scale, the effect of heterogeneity causes a smear of the sharp BuckleyLeverett front (figure 2), as previously noted by Langlo and Espedal [3]. We will show that the value of the mobility ratio across the front seems to play a crucial role in the behavior of mean saturation profiles in heterogeneous porous media.

For $\mathrm{M}_{\mathrm{f}}<1$, the transition zone formed by mean saturations between 0 and $S_{f}$ tends to stabilize and keep a fixed form during the displacement. Every saturation in this zone travels at a constant speed equal to the Buckley-Leverett front's speed : the characteristic lines are straight and parallel in this zone. Hence, the shape of this zone doesn't change in the coordinate system moving at the "front" speed (figure 2b). For saturations greater than $S_{f}$, profiles are close to the rarefaction zone obtained in a homogeneous medium, and are convectively deformed while flooding.

For $\mathrm{M}_{\mathrm{f}}>1$, the whole profile is continuously deformed (figure 2c). All saturation planes follow straight characteristic lines, but each saturation plane speed is different, leading to this stretching

$8^{\text {th }}$ European Conference on the Mathematics of Oil Recovery - Freiberg, Germany, 3 - 6 September 2002 
effect. This is most evident when plotting profiles in the reference frame moving with a saturation between 0 and $S_{f}$ : the behavior of the frontal zone is then completely different than in the stable cases (figure 2d). For saturation well behind the frontal zone, profiles tend to be of Buckley-Leverett type.

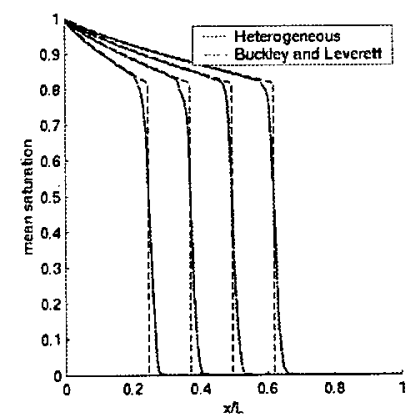

(a)

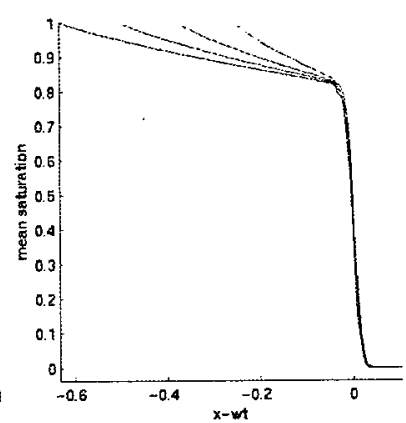

(b)

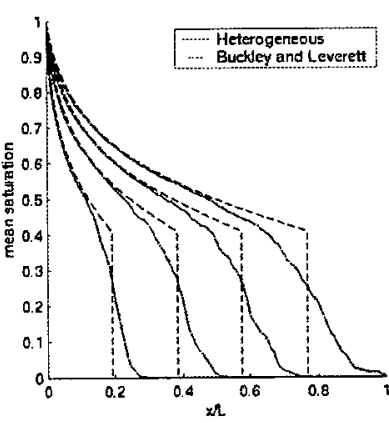

(c)

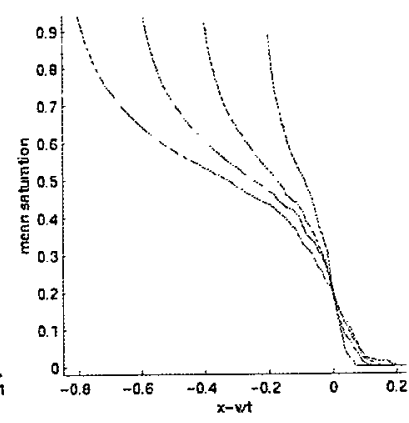

(d)

Figure $2:$ (a) Comparison of mean profiles and B-L profiles. Stable case $M=1 / 2 . \sigma_{\ln (K)}=0.4$ (b) Stable profiles in the front reference frame. (c) Comparison of mean profiles and $B-L$ profiles. Unstable case $M=5 . \sigma_{\ln (K)}^{2}=0.4$ (d) Unstable profiles in the front reference frame.

\section{Modeling mean profiles}

In this section, we search for the large-scale equations governing mean profiles. We try to account for the heterogeneity effects via a dispersive term and a modified convective term in the saturation equation. This approach was previously used by Lenormand [4] for miscible flows in heterogeneous porous media.

The mean saturation profiles obtained in stable cases exhibit the same pattern as profiles observed by Terwilliger et al. for two-phase flows in homogeneous media with capillary forces. Those authors show that the dispersive effect of capillary forces tends to create a stationary capillary zone. The stationary transition zone we obtained is analogous to this capillary zone, although it is only due to the effects of heterogeneity. We can then assume that the effect of small-scale heterogeneity is a dispersive flow at a larger scale, responsible for the formation of the stationary zone. A macrodispersion term $D$ can be introduced in Darcy's laws, leading to a parabolic large-scale equation for mean saturation :

$$
\frac{\bar{u}}{\phi}\left(\frac{d \varphi}{d \bar{S}} \frac{\partial \bar{S}}{\partial x}+\frac{\partial}{\partial x}\left(\psi \frac{\partial \bar{S}}{\partial x}\right)\right)+\frac{\partial \bar{S}}{\partial t}=0
$$

where $\bar{u}$ is the mean total velocity (given by the injection rate) and :

$$
\varphi=\frac{1}{1+\frac{k_{r 2} \mu_{1}}{k_{r 1} \mu_{2}}}, \psi=-\frac{D}{\bar{u}}
$$

are the convective and dispersive parts of the effective fractional flow $: \bar{f}(\bar{S})=\varphi(\bar{S})+\psi(\bar{S}) \frac{\partial \bar{S}}{\partial x}$.

Based upon Terwilliger's observations [5] and the models proposed by, among others, Le Fur [6], we have developed a semi-analytical solution for the macrodispersion equation. This allowed us to realize good fits of the saturation profiles in stable cases (figure $3 a$ ) and to determine the evolution of $D$ with the heterogeneity and the viscosity ratio (figure $3 \mathrm{~b}$ ). Results show a quasi- 
linear evolution of $D$ with $\sigma_{l n(K)}^{2}$, for a given viscosity ratio, except for large variances. D increases with the viscosity ratio. D seems constant for $S_{f}>\bar{S}>0$, while it should depend on $\bar{S}$ for $\bar{S}>S_{f}$ to better fit the remaining of saturation profiles.

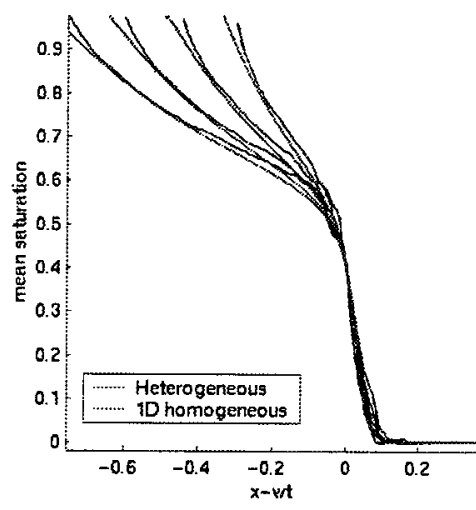

(a)

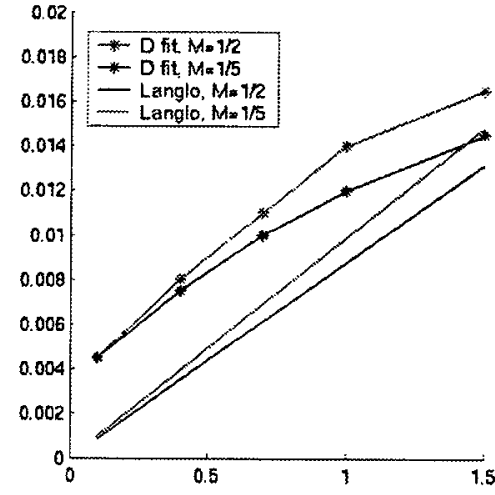

(b)

Figure $3:$ (a) Mean profiles fitted with a macrodispersion description. $M=1 / 2, \sigma_{\ln (K)}=0.4$ (b)Evolution of $D$ with $\sigma_{\ln (K)}^{2}$. Comparison of our fits with the predictions of Langlo et Espedal (equation 7).

For unstable cases, the introduction of a dispersive term is not sufficient because the frontal zone is continuously stretched. The large-scale fractional flow should then be such that it includes both a dispersive term and a modified convective term, this last one representing the fact that each mean saturation $\bar{S}$ travels at a different speed.

The convective stretching of mean profiles is due to the development of a viscous fingering during the flood. Such a behavior was previously analyzed by Koval [7] for miscible flows. Modifying the convective part of the fractional flow is then a way to account for viscous fingering at the larger scale.

However, the introduction of a modified convective part for the fractional flow is not sufficient to completely fit the mean saturation profiles, and it is necessary to add a macrodispersive term $D$ in the description. We then can obtain good fits of the mean profiles (figure 4). As observed previously for stable cases, a constant $D$ seems sufficient for saturations between 0 and $\mathrm{S}_{\mathrm{f}}$, but a saturation-dependent $D$ should be introduced to better fit zones with higher saturations.

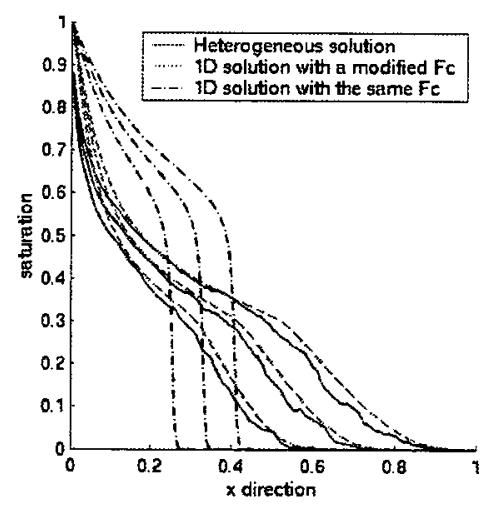

Figure $4:$ Fits of mean profiles in the unstable case $M=5, \sigma_{l n(K)}^{2}=0.4$. Comparison between profiles obtained with an added macrodispersion term only and profiles obtained with a modified convective fractional flow and a macrodispersion term. 


\section{Discussion}

Our results raise two points :

- The front zone's behavior depends on the mobility ratio evaluated at the front. When $M_{f}<1$, a stabilizing process tends to create a finite-length frontal zone, while waterfloodings with $M_{f}>1$ are strongly affected by fingering, with no stabilization of the frontal zone.

- At a larger scale than that of heterogeneity, the mean saturation follows a macrodispersion equation. The convective part of the fractional flow (depending on the $\mathrm{k}_{\mathrm{r}}$ curves) may have to be modified to account for instability.

In the remaining of this paper, we will try to understand and compare these points with previously published theories.

While stochastic methods have largely been being used for several decades for single-phase flows in heterogeneous porous media, they have been less employed for two-phase flows, due to the high non-linearity inherent to multiphase flows. Two kinds of non-linearity arise.

- First, the saturation equation is of hyperbolic type, leading to the building of both a saturation shock and a "rarefaction" zone where saturation profiles are convectively stretched.

- Second, the saturation equation is coupled to the pressure equation via the mobility terms. Hence, a modification of the saturation map will modify the whole velocity map. This phenomenon can be responsible for the development of stable or unstable flows. Velocities become time-dependent, a phenomenon that does non occur in solute transport problems.

This last non-linearity is by far the most challenging and previous attempts to homogenize twophase immiscible flows in heterogeneous porous media have relied on rough approximations to neglect the coupling. Some methods have been developed for steady-state [8] or quasi-static [9] cases where the velocity field is fixed, but very few approaches can cope with transient flows. So far, two major approaches exist : a Lagrangian one, developed by Dagan et al. [10] and Zhang et al. [11], and an Eulerian one, followed by Langlo et Espedal [3]. Both methods rely on the assumption of a fixed velocity field (no coupling between saturation and velocity maps).

In the Lagrangian approach, the velocity field is supposed to be fixed and equivalent to the result of a tracer simulation. Saturations move along fixed streamlines, following the hyperbolic equation along their paths. This allows to cope only with the first kind of non-linearity we described earlier. The problem is hence simplified in two independent steps : the first one consists in relating the streamlines' statistical properties to the permeability field's statistical properties, as in solute transport theory, and the second one is the description of the purely convective displacement of saturations along the fixed streamlines. This approach was first followed by Dagan and Cvetkovic [10], and was extended by Zhang et al [11]. Those authors' results predict a $\sigma_{x}^{2}\left(t, S=S_{f}\right)$ growing with travelled distance, the frontal zone on mean saturations profiles being hence continuously stretched during the flood. The results of Monte-Carlo simulations with fixed velocity fields compare well with these predictions, as shown by Zhang et al. [11], but are completely different from what is actually observed when the full coupling between the velocity field and the saturation map is accounted for, and are similar to the results of a tracer flow, for which the velocity field is always fixed (figure 5).

The viscous coupling between the saturation field and the velocity field is then of crucial importance in the stabilization process of $\sigma_{x}^{2}\left(t, S=S_{f}\right)$, and should be implemented in an upscaling model to correctly model mean saturation profiles. During the flood, the "crossflow" induced by velocity updating in a heterogeneous field is the physical process that damps heterogeneity- 
induced fingers and stabilizes the front when $M_{f}<1$. When $M_{f}>1$, the velocity updating let fingers travel faster, leading to the observed instability.

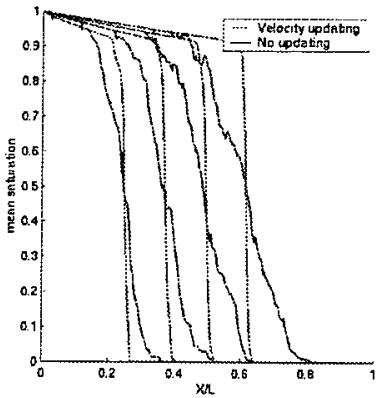

(a)

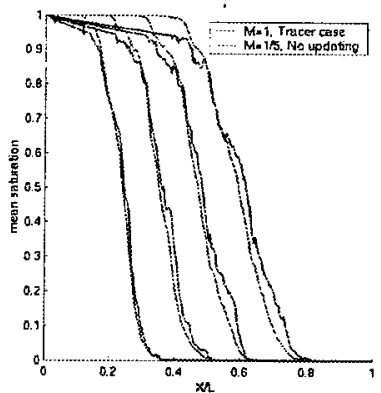

(b)

Figure $5:$ Influence of the velocity field updating. Stable case $M=1 / 2$.(a) Comparison between solutions obtained with and without updating.(b) Comparison with a tracer test result.

The Eulerian approach is based on perturbation developments of the Eulerian flow equations, after splitting all variables in a sum of their mean plus perturbation. Averaging the developed equations leads to an expression for the mean flow, which involves covariance terms. Equations for fluctuations can be multiplied by other fluctuations and averaged, leading to a system of equations for the covariance terms. Langlo and Espedal, 1994, simplify the system to obtain a saturation equation at large-scale, which contains a non-local macrodispersive term taking into account the effects of heterogeneity. This term can be expressed asymptotically by :

$$
D(\bar{S})=\bar{u} \widetilde{\varphi}^{\prime}(\bar{S})^{2} \sigma_{\ln (K)}^{2} I
$$

and the saturation equation at large-scale is :

$$
\frac{\bar{u}}{\phi}\left(\frac{d \tilde{\varphi}}{d \bar{S}} \frac{\partial \bar{S}}{\partial x}+\frac{\partial}{\partial x}\left(D \frac{\partial \bar{S}}{\partial x}\right)\right)+\frac{\partial \bar{S}}{\partial t}=0
$$

where $\widetilde{\varphi}(S)$ is the convex hull of $\varphi(S)$ formed with Welge's tangent, and $I$ is the integral scale of the $K$ field.

This macrodispersive term is obtained after relating the saturation-velocity covariance term to the permeability auto-covariance term with the analytical expression available for tracer cases. The model of Langlo and Espedal is then based on the independence of the total velocity field upon the saturation. Under this assumption, the statistical properties of the velocity field are the same as for a tracer flow.

The macrodispersion parameters $D$ we fitted for stable cases, with a convective fractional flow equal to $\varphi(S)$, following the approach described in the previous section, compare well with Langlo and Espedal's asymptotic predictions for low variances, within about a constant term, certainly due to a small amount of numerical diffusion in our simulations (figure $3 \mathrm{~b}$ ).

However, the model of Langlo and Espedal cannot lead to a stationary frontal zone because the convective fractional flow at large-scale $\tilde{\varphi}(S)$ is a straight line for $S<S_{f}$. Hence, in this formulation, there is no competition between the hyperbolic effect of $\varphi(S)$ that tends to build a shock and the macrodispersion effect of $D$ that tends to stretch the front, so the frontal zone is continuously stretched. Moreover, these authors use perturbation developments of the saturation in the vicinity of the front line, which certainly leads to erroneous results because the saturation experiences a sharp shock across this line. 
These results show that theories based on the hypothesis of no-coupling between the velocity field and the saturation field predict a frontal zone stretched during the flood in a similar way to a tracer test. When the whole non-linearity is taken into account, the velocity field updating causes a flow in the transverse direction to the main flow, which tends to damp fingers when the mobility ratio across the shock is favorable. When this ratio is unfavorable, the velocity field is updated in a way such that fingers travel faster, leading to an unstable saturation field.

\section{Conclusion}

Our study shows that immiscible flows in heterogeneous porous media are strongly affected by the non-linear effects due to viscous coupling. The mobility shock across the saturation front plays a crucial role. In particular, the development of a stationary finite-length frontal zone when $M_{f}<1$ cannot be explained without invoking these non-linear effects. The detailed study of the front dynamics, taking the viscous coupling into account, is possible via a first-order stability analysis. It should relate the front line's statistics to the permeability field's statistics. This study, beyond the scope of this paper, is under progress, and has already shown very promising results.

Acknowledgments : The authors wish to thank Dr Lenormand and Pr Delay for many fruitful discussions. This work is funded by the Institut Français du Pétrole and the Centre National de la Recherche Scientifique.

\section{References}

[1] J. Barker and S. Thibeau, A critical review of the use of pseudo relative permeabilities for upscaling, SPE 35491, 1996.

[2] J. Hagoort, Displacement stability of water drives in water-wet connate-water-bearing reservoirs, SPE 4268, 1974.

[3] P. Langlo and M. Espedal, Macrodispersion for two-phase, immiscible flow in porous media, Advances in Water Resources 17, 297-316, 1994.

[4] R. Lenormand, Determining flow equations from stochastic properties of a permeability field : the M.H.D. model, SPE Journal, 179-190, 1996.

[5] P. Terwilliger, L. Wilsey, H. Hall, P. Bridges, R. Morse, An experimental and theoretical investigation of gravity drainage performance, T. AIME 192, 285-296, 1951.

[6] B. Le Fur, Influences de la capillarité et de la gravité sur le déplacement non-miscible unidimensionnel dans un milieu poreux, Journal de Mécanique, 1(2), 1962, 213-232.

[7] E. Koval, A method for predicting the performance of unstable miscible displacement in heterogeneous media, SPE Journal, 145-154, 1963.

[8] S. Ekrann and J.O. Dahle, Steady-state Upscaling, Transport in Porous Media 41, 245-262, 2000.

[9] M. Quintard and S. Whitaker, Two-phase flow in heterogeneous porous media : The method of large-scale averaging, Transport in porous media (3), 357-413, 1988.

[10] G. Dagan and V. Cvetkovic, Reactive transport and immiscible flow in geological media, Proc. R. Soc. London (452), 285-328, 1996.

[11] D. Zhang, L. Li and H. Tchelepi, Stochastic formulation for uncertainty assessment of two-phase flow in heterogeneous reservoirs, SPE 51930, 1999. 